\title{
A NOTE ON THE STRATEGY OF BREEDING CLONAL CROPS
}

\author{
N. W. SIMMONDS \\ Edinburgh School of Agriculture, West Mains Road, Edinburgh, EH9 3JG, Scotland
}

Received 27.ii.84

\section{SUMMARY}

It is shown that, in breeding a clonal crop for several diverse environments, it is probably best to select populations in those environments and exchange clonal material extensively at an early stage. An example is drawn from sugarcane. There is evidence in this crop that selection is rather inefficient and that superior genotypes may occur at higher frequencies than would usually be thought likely.

\section{InTRODUCTION}

This note was prompted by consideration of the strategy of sugarcane breeding in the West Indies. There is a central station in Barbados which makes the crosses and distributes seed to five widely separated breeding stations. The five stations raise seedlings, select over generations among the clonal progeny and isolate potential varieties for intensive trial. The whole programme operates on an "all for one and one for all" basis. The question obviously arises: Is it better to exchange clones at the "finished" variety level or at some earlier stage?

\section{THEORY}

In a clonal crop, suppose we have a breeding programme of fixed size, $4 N$ plants, distributed equally between four generations or phases. A new set of $N$ seedlings is planted each year and we assume a constant selection rate $\alpha$ (Finney, 1958). Falling numbers of clones is approximately compensated by increased numbers of plants per clone so that each phase is of roughly constant size, $N$. From stage 4 there emerges a batch of clones deemed worthy of extended trial. If a good clone is present in the sample, the probability of its retention, $k$, will start low and rise through the generations and the product of the $k$ over generations $(m)$ will be the overall probability of retention to the end. We do not know the $k$ but can assume they start low (maybe not much greater than $\alpha$ ) and approach unity at the end of phase 4. Four different assumptions about values of $k$ are made in table 1. Neither they nor the assumption of constant $\alpha$ is critical for the main argument. Finney (1958) shows that constant $\alpha$ and constant resources per phase are roughly optimal.

Now suppose that the chance that any random genotype is excellent is $f$ so that excellent clones occur with mean per plant frequency $f$ and in number $N f$ in the first generation. The mean number of excellent clones produced by the programme in any year is therefore $m f N$ and this might be in the range $0.04 f N$ to $0.39 f N$ on the assumptions of table 1 .

Now suppose that there exists another programme, equal in size, the same in structure and in a similar (though not identical) environment. The 
TABLE 1

Structure of programme; $N$ plants per generation at constant selection rate, $\alpha ; k$ is the chance of retaining an excellent clone, $m$ the cumulative chance

\begin{tabular}{ccccccc}
\hline Year & Plants & Clones & \multicolumn{4}{c}{$k$ assumed for four cases } \\
\hline 1 & $N$ & $N$ & 0.60 & 0.60 & 0.20 & 0.20 \\
2 & $N$ & $N \alpha$ & 0.78 & 0.69 & 0.58 & 0.39 \\
3 & $N$ & $N \alpha^{2}$ & 0.87 & 0.78 & 0.76 & 0.58 \\
4 & $N$ & $N \alpha^{3}$ & 0.95 & 0.95 & 0.95 & 0.95 \\
\cline { 3 - 7 } & $4 N$ & & $m=0.387$ & 0.307 & 0.084 & 0.043 \\
\hline
\end{tabular}

two programmes agree to exchrange material at the end of phase 2 so that phases 3 and 4 are each doubled in size and phases 1 and 2 correspondingly reduced. Then $N^{\prime}+N^{\prime}+2 N^{\prime}+2 N^{\prime}=4 N$ and $N^{\prime}=2 N / 3$. Assume that a good clone is equally likely to be retained in either programme up to the end of stage 2 , that is, that the environments are non-discriminatory at the earlier levels of selection. The mean success of each programme will then be $1.333 \mathrm{mfN}$ or $0.516 f N, 0.409 f N, 0.120 f N$ and $0.057 f N$ under the four assumptions as to $m$. Results for each programme are thus enhanced by a factor of $1 \cdot 333$, independently of the size of $m, f$ and $N$.

Next, suppose that the two programmes went their own ways, did not exchange numerous clones at stage 3 but did exploit each other's "finished" clones emerging from trials following stage 4 . Assume a probability, $r$, that a clone finally successful in one place will also be successful in the other. The mean success for each programme will then be $(1+r) m f N$ and, at breakeven point, $(1+r) m f N=1.333 m f N$, whence $r=0.333$. Thus, if $r<$ 0.333 , it would be reasonable to exchange clones early but not if $r>0.333$. More generally, for $n$ programmes, rates of success are $(1+(n-1) r) m f N$ if the programmes remain separate and $\{2 n /(n+1)\} m f N$ if they operate jointly, with breakeven $r=1 /(n+1)$. For five programmes, $N^{\prime}=N / 3$ and breakeven $r=0 \cdot 167$. In general, then, it would be best to maximise initial seedling populations and exchange only "finished" varieties if genotypes were widely adapted ( $r$ large); but to reduce seedling numbers and exchange clones early if varietal adaptation were narrow ( $r$ small).

TABLE 2

Results of exchange for varying numbers of programmes, $n ; r_{b}$ is $r$ at breakeven point

\begin{tabular}{clllll}
\hline$n$ & 1 & \multicolumn{1}{c}{3} & 3 & 4 & \multicolumn{1}{c}{5} \\
$1+(n-1) r$ & 1 & $(1+r)$ & $(1+2 r)$ & $(1+3 r)$ & $(1+4 r)$ \\
$2 n /(n+1)$ & 1 & 1.333 & 1.500 & 1.600 & 1.667 \\
$N^{\prime}$ & $N$ & $0.667 N$ & $0.500 N$ & $0.400 N$ & $0.333 N$ \\
$r_{b}$ & - & 0.333 & 0.250 & 0.200 & $0 \cdot 167$ \\
\hline
\end{tabular}

The condition that the four phases (at constant total effort, $4 N$ ) are of approximately equal size may be relaxed. If phases 3 and 4 are in the ratio $s$ to phases 1 and 2 , then $r$ at breakeven becomes $1 /(s n+1)$. Even large changes in $s$ (say $0.5,1.5$ ) make relatively little difference to $r_{b}$ (recalling that $r$ is a very imperfectly known quantity). Obviously, large $s(>1)$, 
implying costly later phases, makes for low $r_{b}$ and tends to disfavour early exchange.

\section{EXAMPLE AND DISCUSSION}

The West Indies Central Sugarcane Breeding Station is in Barbados and there are five testing stations (so-called, they are really breeding stations) in: Guyana, Trinidad, Barbados, Jamaica and the Dominican Republic. In the 1930s and 1940s the (then) British West Indies were served by a single programme in Barbados and the B-canes were widely successful, not only in the West Indies but in Latin America and indeed elsewhere. It was noteworthy that few canes were really widely adapted and that those that did well in one place were not very likely to do well elsewhere. A considerable degree of local agro-ecological adaptation was thus evident, with the corollary that local selection $a b$ initio in each place would be sensible. The conclusion was strengthened by the observation that Guyana has long had a modest breeding programme which has produced some excellent local (D) canes which have never been successful in other places; it is not notably weakened by the existence of one exceptionally widely adapted cane (HJ 5741) selected before this programme began.

In the late 1950s, therefore, the present dispersed programme was initiated and the four ex-British stations were joined by Central Romana (Gulf and Western Corporation, Dominican Republic). Central Romana also has a substantial domestic programme (separate from the joint one) the results of which are not included in the following analysis. The intention, from the start of the dispersed programme, was for all five members to exchange numerous selections at a fairly early stage in order more fully to exploit the genetic potential by minimising the loss of canes potentially excellent in one place being discarded through selection in a different environment. In practice, the rate of exchange has been very low, largely due to plant quarantine difficulties. My calculations suggest that the consequent loss may have been considerable.

To consider some numbers (Walker, pers. comm.), total seedlings raised in the period $1957-1977$ were $2 \cdot 184 \times 10^{6}$ (a small programme by sugarcane standards: Walker and Simmonds, 1984). Yearly and station productions were (and are) somewhat erratic, with averages $104 \times 10^{3}$ per year and $20.8 \times 10^{3}$ per station-year. Selection patterns within stations vary but I assume that the pattern of table 1 and an average throughput of $20.8 \times 10^{3}$ are roughly applicable. Successes have been about 12 clones commercially successful or expected soon to be so; they are unevenly distributed, from zero to six per station and none has (yet) succeeded in any other of the four places (though several have done so outside the five). The average rate of success per station-year is $m f N=0.114$ so, with $N=20.8 \times 10^{3}$ and $m$ guessed in the range 0.04 to 0.39 (table 1 ), $f$ would be in the range $14 \times 10^{-6}$ to $140 \times 10^{-6}$. I think a low value of $m$ is most likely so that $f$ might be about $50 \times 10^{-6}$ (but could be higher, even much higher-see below).

As to the size of $r$, it was probably (on the basis of commercial variety lists supplied by $\mathrm{Mr} \mathrm{D}$. I. T. Walker) about $0 \cdot 2-0 \cdot 5$ several decades ago when there was little or no competition; even now, in places where there are no local breeding programmes (Caribbean islands and certain Latin American countries) B-canes (often different from those that succeed in 
Barbados) do well. Within the five member-countries, however, all the signs are that $r$ is now low: local selection succeeds best. I stated above that exchange has, in practice, been small, indeed virtually negligible. From the theory above, it looks as though full exchange would have reduced the annual seedling throughput from $20.8 \times 10^{3}$ to about $7 \times 10^{3}\left(N^{\prime}=N / 3\right)$ but increased the successes from about 12 to about $17(r=0.1)$ or $20(r=0)$ varieties. There is another argument in favour of exchange, namely speed. Exchanged clones could enter other programmes with a delay of a year for multiplication. Clones exchanged only at the variety level still have to be locally tested, adding about five years. So there would be (roughly) a four-year advantage to early exchange.

In practice, unfortunately, it seems likely that quarantine will always be an insuperable obstacle to adequate exchange between politically separate places (though the principle would remain good within a unified continental territory). Even if exchange were infeasible, however, the calculations draw attention to another feature of efficiency, namely the size of $m$ and the critical nature of the first round of selection. If $m$ is really about $0 \cdot 1$, some 90 per cent of the best clones are being lost. There is, indeed, evidence that losses are high, that is, that selection is inefficient. Thus Young Kong (1973) found that a random sample of seedlings, though on average somewhat inferior to selected samples, still contained about 5 per cent of clones presumptively of the same order of performance in sugar yield as the standard, implying $f$ in the order of one in a few thousand after selection for secondary characters. MacColl's (1977) studies of random samples of seedlings in comparison with parents showed that, although family means were all below mid-parents, three families out of four gave evidence of fair frequencies of clones equal to or better than their better parents and the best family contained about 5 per cent of progeny at least equal to the mean of the two best clones in the experiment. Meyer, Heinz and Wu (1982) compared first and second choice selections with random sample and, after another round of selection, could detect no difference between the three groups in terms of sugar yields in trials against standards: each contained about 35 per cent of clones not demonstrably worse than standards. Selection had thus been quite ineffective and superior clones were remarkably frequent ( $f$ large). And, again, Walker (1981, pers. comm.) compared the products of first and second choice selections with standards and found no difference in sugar yields between the selected groups (there was no random sample).

Jointly, these results do not encourage a belief in even moderate efficiency of selection, so $m$ is probably small and, as a corollary, $f$ large. If so, overall economy might well be served by reducing $N$ and relaxing selection very greatly, whether or not exchange were practised. There have been similar doubts about the efficiency of selection in potatoes but in no crop, I think, have good estimates of $m$ and $f$ been made; it would be difficult but well worthwhile to get them.

Acknowledgement. I thank the referee for helpful criticism.

\section{RefERENCES}

FINNEY, D. J. 1958. Plant selection for yield improvement. Euphytica, 7, 83-106.

MACCOLL, D. 1977. Growth and sugar accumulation in sugarcane III. Expl Agric., 13, 161-167. 
MEYER, H. K., HEINZ, D. J. AND wU, K. K. 1982. Visual selection II. Hawaiian Sug. Pltrs Ass. ann. Rep., 1982, 8-9.

WALKER, D. I. T. AND SIMMONDS, N. W. 1984. Sugarcane breeding. In: Sugarcane, Blackburn, F. H., Longman, London.

YOUNG KONG, V. M. 1973. Comparison of the efficiency of three methods of selection in a mixed seedling population. Proc. West Ind. Sug. Cane Technol., Barbados, 1973, 176-181. 